\title{
Evidence for giant interacting coronal streamers in a pre-main-sequence binary system
}

\author{
Maria Massi* \\ Max Planck für Radioastronomie \\ E-mail: mmassi@mpifr-bonn.mpg.de
}

\begin{abstract}
Here we report on the VLBI discovery of solar-like extended streamers anchored on the two weak-line T Tauri stars of the binary system V773 Tau A. Covering the interbinary distance the 20 stellar radii extended streamers enter in collision during each stellar rotation with consequent occurrence of magnetic reconnection. Thermal electrons confined in the streamers become accelerated to relativistic speeds and emit synchrotron emission in the radio band making the magnetic streamers "visible" in the VLBI images. This is different from the solar case where the emission from the streamers is just scattered photospheric light that would never be observable in distant objects. Evidence of extended solar-like streamers in T Tauri stars, thought to be fully convective, or nearly fully convective objects, indicates that the tachoclinal layer, in this case either not existing at all or buried very deeply, is not relevant for the formation of such solar-like magnetic structures.
\end{abstract}

The 9th European VLBI Network Symposium on The role of VLBI in the Golden Age for Radio Astronomy and EVN Users Meeting

September 23-26, 2008

Bologna, Italy

\footnotetext{
* Speaker.
} 


\section{Introduction}

Coronae are a phenomenon present among a wide variety of objects [1]. Coronae exist not only in solar-like late type stars, but also young objects, not yet in main sequence, show highly elevated levels of coronal activity and even accretion disks, around both compact objects and protostars, are postulated as well to have a corona. From X-ray observations the solar corona results to be formed by closed bright (i.e. trapping dense and hot plasma) magnetic field lines, called coronal loops and open magnetic field lines appearing as dark areas, called coronal holes through which the fast (400-800 km/sec) solar wind flows [2]. At the top of the closed coronal loops, extended streamers are observable in visible light during total solar eclipses, and with satellite-borne coronagraphs as in the Solar and Heliospheric Observatory Large Angle and Spectrometric Coronagraph Experiment (SOHO LASCO). In the observed radiation, that is the result of the Thomson scattering of photospheric light, one sees a helmet, i.e. a cusp-shaped field line located on top (2 to 4 $R_{\odot}$ ) of a closed coronal loop, and a very long streamer which extends out to $30 R_{\odot}$. Through the solar helmet streamers flows the slow wind (up to $400 \mathrm{~km} / \mathrm{s}$ ) [2-4]. During the solar activity cycle the structure of the corona dramaticaly changes. During sunspot minimum high-latitude open field lines form the familiar polar coronal holes, whereas meridional closed coronal loops form around the equator. Wind and solar rotation force open magnetic field lines from the two hemispheres above the closed loops forming the typical helmet streamers belt around the equator. As the solar activity increases the geometry and orientation of the closed structures complicates. During the maximum activity closed coronal loops pratically parallel to the equator appear at all latitudes and helmet streamers are located everywhere, even over the Sun's poles [5]. The solar corona itself is therefore already higly structured and dynamic, to what extent can we apply our knowledge on the Sun to other coronae?

The theory of dynamo developed by Parker [6] explains how the differential rotation generates a toroidal field in the interior of the sun in the transitional layer, called the tachocline, between two distinct rotational regimes: the differentially-rotating solar convective zone and the radiative interior where the rotation is uniform [7]. The corona's building block, i.e. the coronal loop, is created by the emersion to the surface by magnetic buoyancy of small portion of flux tubes of this toroidal field. Very young stellar objects, however, are thought to be nearly fully convective, or fully convective, so the tachoclinal layer is either buried very deeply, or does not exist at all. An important open issue is therefore if different physics of magnetic field generation may determine large differences in the resulting corona and if fully convective objects can have large scale solar-like magnetic fields [8]. Here we report on a research based on radio observations with the Effelsberg 100-m telescope, the Plateau de Bure Interferometer "PdB", the Very Large Array "VLA" and the Very Long Baseline Array "VLBA". The target were two pre-main sequence objects: the two weak-line $\mathrm{T}$ Tauri components of the system V773 Tau A.

\section{The system V773 Tau A}

The system V773 Tau (HDE 283447) at a distance of 136 \pm 5 pc [9], in the Taurus-Auriga starforming region, is a quadruplet of young stellar objects within an area of radius less than $100 \mathrm{AU}$ (700 mas): the A component, our target, is a double-lined spectroscopic binary of two weak-line T 


\begin{tabular}{lccc}
\hline & & Aa(Primary) & Ab (Secondary) \\
$\operatorname{MasS}^{[9]}$ & $(\mathrm{M} \odot)$ & 1.54 & 1.33 \\
$\operatorname{Radius}^{[9]}$ & $\left(\mathrm{R}_{\odot}\right)$ & 2.22 & 1.74 \\
$\mathrm{P}_{\text {rot }}^{[18]}(\mathrm{d})$ & & 2.5 & 1.9 \\
\hline
\end{tabular}

Table 1: The binary system V773 Tau A

Tauri stars ( $\mathrm{Aa}$ and $\mathrm{Ab}$ ), the $\mathrm{B}$ and $\mathrm{C}$ components, with significant infrared excess, are at an earlier evolutionary stage. The two components V773 Tau Aa and Ab (Table 1), with orbital period of $51.1033 \mathrm{~d}$ and orbital separation $(2 a)$ of 5.5. mas ( $0.8 \mathrm{AU})$, are still in the quasi-isothermal vertical track (contraction phase) and have an estimated age of $3 \pm 1$ Myr [9].

\section{Interbinary collisions}

V773 Tau A was observed with the Effelsberg 100-m telescope over a frequency range spanning from $8 \mathrm{GHz}(3.6 \mathrm{~cm})$ to $40 \mathrm{GHz}(7 \mathrm{~mm})$ for 522 days during which where collected 110 samples. The source was also observed with the VLA for one month at a frequency of $14.96 \mathrm{GHz}$ $(2.0 \mathrm{~cm})$. Figure 2 in Massi, Menten and Neidhöfer [10], presents the data folded with the orbital period ( $51 \mathrm{~d}$ ) and reveals that the system exhibits persistent flaring activity that gradually increases from few mJy at apoastron to very strong $(S \sim 100 \mathrm{mJy})$ flares around periastron.

There exists a close relationship between flares and interaction of magnetic structures. In fact, as observed on the Sun, flares can be triggered by interactions between new and older emergences of magnetic flux in the same area [11] i.e. when in its emergence, because of magnetic buoyancy, on the surface, one coronal loop happens to intrude into other already established coronal loop, magnetic reconnection occurs. Part of the magnetic energy released during reconnection goes to accelerate a fraction of the thermal electrons trapped in the flaring loop and a population of relativistic electrons is produced [12]. These relativistic particles gyrating around the magnetic field lines of the coronal loop, where they are confined, generate synchrotron emission in the radio band. Applying this knowledge of solar flares to V773 Tau A, it is clear that in this system the observed relationship between intensity of the flare occurrence and distance of the two stars indicates another, new mechanism of magnetic interaction, that of interacting coronae and therefore with magnetic reconnection taking place far out from the stellar surfaces, where the two coronae interact with each other. Knowing that the distance between the two stars at apoastron and at periastron is $52 R_{*}$ and $30 R_{*}$, respectively, interbinary collisions would then imply very large coronal structures with a size of $15 R_{*} \leq H \leq 26 R_{*}$. As a matter of fact a large magnetic structure has been imaged by using Very Long Baseline Interferometry [13] showing a large halo ( $\geq 3$ mas, $\sim 40 R_{*}$ ) surrounding two compact components separated 1.14 mas $\left(\sim 16 R_{*}\right)$. On other hand Skinner et al. [14] interpreted the light curve of a hard X-ray flare in V773 Tau A as being due to the rotational modulation of the emitting flaring region, determining a size of $H \leq 0.6 R_{*}$. Tsuboi et al. [15] interpreted the decay of another hard X-ray flare as being due to radiative cooling, obtaining a size of $1.4 R_{*}$. Therefore, radio and X-ray emission clearly come from spatially separated regions: smaller ones those associated with X-ray emission and larger ones those associated with radio emission. Further confirmation of the presence of two structures comes from the multiwavelength campaign on V773 


\begin{tabular}{ccccc}
\hline Run & $\begin{array}{c}\text { Date } \\
(\mathrm{JD})\end{array}$ & $\Phi$ & $\begin{array}{c}\mathrm{Ha}-\mathrm{Hb} \\
(\mathrm{mas})\end{array}$ & $\begin{array}{c}\mathrm{Aa}-\mathrm{Ab} \\
(\mathrm{mas})\end{array}$ \\
\hline A & 2453076.34 & 0.32 & & 2.7 \\
B & 2453077.34 & 0.34 & 2.8 & 2.9 \\
C & 2453078.34 & 0.35 & 3.2 & 3.1 \\
D & 2453079.34 & 0.37 & 3.2 & 3.2 \\
E & 2453080.34 & 0.39 & 3.4 & 3.3 \\
F & 2453081.34 & 0.41 & 3.5 & 3.4 \\
G & 2453082.34 & 0.43 & 3.1 & 3.5 \\
\hline
\end{tabular}

Table 2: Stellar and helmet separations in the young stellar binary system V773 Tau A during each VLBA+EB observation. Julian day 2453076.34 corresponds to March 11, 2004 at 20:10. Ha and Hb are the two radio peaks in the VLBI images associated with the helmets. Aa and Ab are the primary (V773 Tau $\mathrm{Aa}$ ) and secondary (V773 Tau Ab) stars of the system respectively. The error associated to the separations is \pm 0.1 mas. At a distance of $136 \mathrm{pc}$, one stellar radius, $R_{*} \simeq 2 R_{\odot}$, corresponds to 0.069 mas.

Tau A carried out by Feigelson et al. [16] showing radio variability combined with a steady X-ray flux. Following the solar analogy we postulated solar-like extended streamers located at the top of the observed stellar sized X-ray emitting coronal loop. V773 Ta A was observed with the IRAM Plateau de Bure Interferometer at $3 \mathrm{~mm}$ and a complete (onset and decay) flare of $360 \pm 17 \mathrm{mJy}$ was observed close to periastron $(\Phi=0.1$ ). The flare decay (e-folding time of $2.31 \pm 0.19$ hours ) was modeled with a slow leakage of relativistic electrons trapped in a magnetic structure with two mirror points: one close to the star (at 2-5 $\mathrm{R}^{*}$ ) i.e. the helmet and the other to the top of the streamer (at 10- $20 \mathrm{R}^{*}$ ) i.e. where the two streamers interact with each other [17]. At the distance of $136 \mathrm{pc}$ [9], one stellar radius $\left(R_{*} \simeq 2 R_{\odot}\right.$ ), corresponds to 0.069 mas. Only the high resolution of VLBI/VLBA can probe the proposed model.

\section{VLBA observations}

\subsection{Interbinary collision of streamers catch in the act}

We observed V773 Tau A for seven consecutive days (listed as A to G in Table 2) at $8.4 \mathrm{GHz}$ with the VLBA together with the Effelsberg 100-m radio telescope (VLBA+EB) [18]. Whereas in the image of the first run there is only one $3 \sigma$ feature, two structures are present in all the other images (see here Fig. 1-B,E,G), here called North-East, NE, and South-West, SW. The two structures can be quite complex as in Fig. 1-B where they show two components each ("a", "b" for the NE and "c", "d" for the SW). The location of the stars in the radio images of Fig. 11, is given as a cross [18]. In Fig. 1 1 -B, the two features are extended $18 R_{*}$ each, which are the expected $H$ size for the postulated streemers. The relativistic particles, produced by magnetic reconnection at the top of the streamer, spiralling around magnetic field lines emit synchrotron radiation and make the whole streamers "visible" in the radio band.

Numerical calculations based on the general equation of radiative transfer show that the distributions of energetic electrons along a solar flare coonal loop are highly inhomogeneous: accelerated electrons are concentrated where the magnetic field is stronger [19]. Radio images, during 
solar flares show brightness peaks located at the footpoints of the coronal loop [20], which are the mirror points of the trapping structure. From that it follows that during a flare the brightness peaks in the radio images reveal the position of the mirror points. Whereas in the solar coronal loop case the mirror points are the footpoints of the loop, in V773 Tau A the two mirror points for each of the two NE and SW features, are one close to the star and the other displaced several stellar radii in the upper corona. The model based on the flare decay of the flare at $3 \mathrm{~mm}$, derived a first mirror point close to the star (at 2-5 $\mathrm{R}^{*}$ ) i.e. the helmet. The peaks "a" and "c", that are the two closest peaks to the two related stars, are therefore the two helmets. The second mirror point in the model is located at the top of the streamer (at 10- $20 \mathrm{R}^{*}$ ) i.e. where the two streamers interact with each other. As a matter of fact the two extremes "b" and "d" of the two features in Fig. 1-B not only are at the predicted distance from their respective helmets, but also are close each other, as one expect from interacting streamers. It seems that in run B (see sketch of the system in Fig. 1) we were lucky enough to catch the interaction of the two streamers in the act.

\subsection{Streamers anchored on rotating stars}

The 100-m telescope monitoring shows (Fig. 3 in [18]) that at the time of image Fig. 1$\mathrm{E}$ the flare is already in its decaying phase. In fact, while a total flux density of $12 \mathrm{mJy}$ was measured with the Effelsberg 100-m telescope two hours before the VLBA+EB observation, only $6 \mathrm{mJy}$ were observed when the observation begun. If the collision occurs when the streamers are aligned as in Fig. 1-B, one expects that few hours ( $>2 \mathrm{~h}$ ) after the collision, because of the short rotation period ( $\simeq 2 \mathrm{~d}$, Table 1$)$ the streamer, anchored on the rotating star, should appear in the image appreciably rotated with respect to the position angle of the streamer in Fig. 1-B $\left(\mathrm{PA}=208^{\circ}\right)$. Indeed the position angle of the NW feature of Fig. 1.E is of PA=273 ${ }^{\circ}$. Besides the rotation, another fact indicates that time has elapsed from the streamer-streamer collision: the absence of the upper mirror point in the SW feature. The solar streamers are open structures. Therefore, one expects that the compressed magnetic field lines at the top of the streamer ("d" and "b") relax back to an open configuration, i.e., the peaks at "d" and "b" should have a faster decay with respect to the helmets "a" and "c". The relativistic electrons diffuse from the relaxing upper mirror points in progressively larger structures: in Fig. 1. B, that is just after/during a collision, the sources are point-like, whereas in Fig. 1 E, showing an already evolved situation, the sources are extended. With this respect, in the last observation of March 17 (Fig. 1-G), the facts that on one hand the helmet streamer anchored on the primary star appears substantially rotated and extended and on the other hand, there is a lack of the upper mirror in the NE structure, suggest again a flare decay scenario as for Fig. 1-E, only applied to the other star.

\section{Conclusions and Discussion}

In our VLBA observations individual streamers can be distingueshed in each of the two stars of the system V773 Tau A. The complete extent of the helmet streamers is the observed value of 18 $R_{*}$, between helmet and upper mirror point, plus the distance, of a few stellar radii, of the helmet itself from stellar surface. The practically permanent flaring activity in V773 Tau A, from a level of a few mJy around apoastron $\left(52 R_{*}\right)$ to more than $100 \mathrm{mJy}$ at periastron $\left(30 R_{*}\right)$ [10] is explained by the interaction of these extended streamers. At the short periastron distance the two coronae 

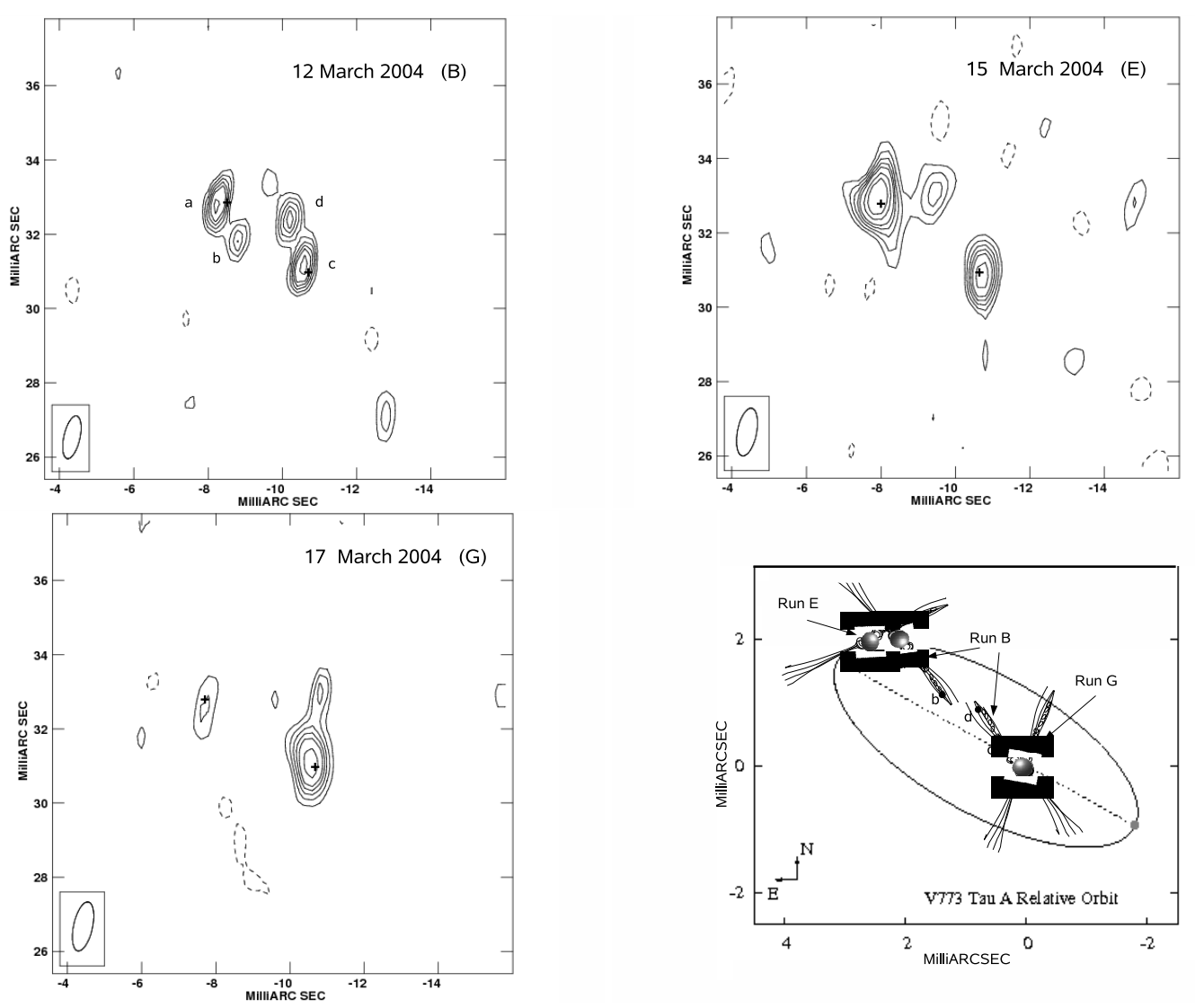

Figure 1: $8.4 \mathrm{GHz}$ VLBA+EB images of the young stellar binary system V773 Tau A. Stellar positions are indicated by crosses [18]. The peak flux density in the three B, E and G images are $0.5,0.7$, and 0.5 $\mathrm{mJy} / \mathrm{beam}$ respectively. Contour levels are $-1,1,1.5,2,2.5,3,4$ and 5 times the $3 \sigma(0.1 \mathrm{mJy} / \mathrm{beam})$. The beam size is shown in the bottom left corner of each panel $(\sim 1.3 \times 0.5$ mas $)$. The sketch of the system shows helmet streamers anchored on rotating stars (not to scale).

nearly overlap, giving rise to the observed giant flares (i.e. up to $360 \mathrm{mJy}$ at $\lambda 3 \mathrm{~mm}$ [17]) and to the large structure imaged by VLBI by Phillips and collaborators [13].

Our observation [18] proves that solar-like streamers of more than $20 R_{*}$ exist also in fully / nearly fully convective objects, that is in objects where the tachocline is missing or buried very deeply. Besides, the helmet streamers are an important magnetic configuration for themselves. Through the solar helmet streamers flows the slow wind (up to $400 \mathrm{~km} / \mathrm{s}$ ). The plasma flow presents density fluctuations, i.e. "blobs" or "plasmoids" that seem to be the product of reconnection or small-scale eruptions at the cusp of the helmet streamer. On other hand helmet streamers are related with coronal mass ejections, where plasma clouds leave the sun in violent way (impulsive CMEs) ([2] and references therein). Because of these characteristics of being associated to plasma motions/ejections and because of their topology, i.e. open field lines pushed above a closed loop, the helmet streamers are invoked as a key magnetic feature bridging the gap between corona and jet. In fact, they have been proposed in the process of jet formation in microquasars/AGN [21] and also in young stellar objects [22,23]. The discovery of helmet streamers in stars other than the Sun, 
at a wavelength observable at high resolution, could therefore lead to a deeper understanding of these important magnetic structures and their possible related processes.

\section{Acknowledgments}

Based on observations with the Effelsberg 100-m telescope of the Max-Planck-Institut für Radioastronomie (MPIfR) and the Very Long Baseline Array (VLBA). The VLBA is a facility of the National Radio Astronomy Observatory (NRAO), operated by Associated Universities Inc. under a cooperative agreement with the National Science Foundation (NSF).

\section{References}

[1] M. Massi and T. Preibisch, Coronae of stars and accretion disks, MmSAI 78 (217) 2007.

[2] R. Schwenn, Solar Wind Sources and Their Variations Over the Solar Cycle, Space Science Rev. 124 (51) 2006.

[3] A. Vourlidas, A Review of White Light Streamers at the End of Cycle 23, in proceedings of Solar Activity and its Magnetic Origin, eds Bothmer, V. \& Hady, A. Cambridge University Press 2006.

[4] S. Suess and S. Nerney, Flow in streamer boundaries, and streamer stability, Advances in Space Research 33 (668) 2004.

[5] S. Bravo, G. A. Stewart and X. Blanco-Cano, The Varying Multipolar Structure of the Sun's Magnetic Field and the Evolution of the Solar Magnetosphere Through the Solar Cycle, Sol.Phys. 179 (223) 1998.

[6] E. N. Parker, Cosmical magnetic fields: Their origin and their activity, Oxford, Clarendon Press, New York 1979.

[7] M. S. Miesch, Large-Scale Dynamics of the Convection Zone and Tachocline, Living Reviews in Solar Physics 2 (1) 2005.

[8] W. Dobler, M. Stix and A. Brandenburg, Magnetic Field Generation in Fully Convective Rotating Spheres, ApJ 638 (336) 2006.

[9] A. F. Boden, G. Torres, A. I. Sargent, R. L. Akeson, J. M. Carpenter, D. A. Boboltz, M. Massi, A. M. Ghez, D. W. Latham, K. J. Johnston, K. Menten and E. Ros Dynamical Masses for Pre-Main-Sequence Stars: A Preliminary Physical Orbit for V773 Tau A, ApJ 670 (1214) 2007.

[10] M. Massi, K. Menten, J. Neidhöfer, Periodic radio flaring on the T Tauri star V 773 Tauri, A\&A, 382 (152) 2002.

[11] M. Nishio, K. Yaji, T. Kosugi, H. Nakajima and T. Sakurai, Magnetic Field Configuration in Impulsive Solar Flares Inferred from Coaligned Microwave/X-Ray Images, ApJ 489 (976) 1997.

[12] Drake, M.A. Shay, W. Thongthai, M. Swisdak, Production of Energetic Electrons during Magnetic Reconnection, Physical Review Letters 94 (95001) 2005.

[13] R. B. Phillips, C. J. Lonsdale, E. D. Feigelson and B. D. Deeney, Polarized Radio Emission From the Multiple T Tauri System HD 283447, ApJ 111 (918) 1996.

[14] S. L. Skinner, M. Guedel, K. Koyama and S. Yamauchi, ASCA Observations of the Barnard 209 Dark Cloud and an Intense X-Ray Flare on V773 Tauri, ApJ, 486 (886) 1997. 
[15] Y. Tsuboi, K. Koyama, H. Murakami, M. Hayashi, S. Skinner and S. Ueno, ASCA Detection of a Superhot 100 Million K X-Ray Flare on the Weak-lined T Tauri Star V773 Tauri, ApJ 503 (894) 1998.

[16] E. D. Feigelson, A. D. Welty, C. Imhoff, et al. Multiwavelength study of the magnetically active T Tauri star HD 283447, ApJ 432 (373) 1994.

[17] M. Massi, J. Forbrich, K. M. Menten, G. Torricelli-Ciamponi, J. Neidhöfer, S. Leurini and F. Bertoldi, Synchrotron emission from the T Tauri binary system V773 Tauri A, A\&A 453 (959) 2006.

[18] M. Massi, E. Ros, K. M. Menten, M. Kaufman Bernado', G. Torricelli-Ciamponi, J. Neidhöfer, A. Boden, D. Boboltz, A. Sargent, G.Torres, Interacting coronae of two T Tauri stars: first observational evidence for solar-like helmet streamers, A\&A 4804892008.

[19] A. H. Zhou, Y. N. Su and G. L. Huang, Energetic Electrons in Loop Top and Footpoint Microwave Sources, SoPh 226 (327) 2005.

[20] T. S. Bastian, A. O. Benz, D. E. Gary, Radio Emission from Solar Flares, ARAA 36 (131) 1998.

[21] E. M. de Gouveia dal Pino, The Role of Magnetic Fields on Astrophysical Jets, in proceedings Magnetic Fields in the Universe: From Laboratory and Stars to Primordial Structures, AIPC 784 (183) 2005.

[22] J. Ferreira, C. Dougados and S. Cabrit, Which jet launching mechanism(s) in T Tauri stars?, A\&A 453 (785) 2006.

[23] E. C. Ostriker and F. H. Shu, Magnetocentrifugally Driven Flows from Young Stars and Disks. IV. The Accretion Funnel and Dead Zone, ApJ 447 (813) 1995. 